\title{
MULTI-GEV ELECTRON LINAC-PULSE STRETCHER DESIGN OPTIONS
}

\author{
R.C. York, J.S. McCarthy, B.E; Norum* \\ Department of Physics \\ University of Virginia \\ Charlottesville, Virginia 22901
}

\section{Abstract}

The Linac-Pulse Stretcher Ring system is evaluated as a design for a high duty factor ( $) 90 \%$ ), intense $(240 \mu \mathrm{A})$, electron accelerator for operation in the 0.5 to multi-GeV (> $4 \mathrm{GeV}$ ) region. Options within this system are discussed in light of their predicted initial and operational cost, flexibility, and reliability. The ramifications of the choice of a particular cavity type, accelerating gradient, repetition rate, beam pulse length, and number of beam recirculations for the linac design are discussed both with respect to the linac itself and to the Pulse Stretcher Ring. The Pulse Stretcher Ring concept is analysed in terms of injection schemes (single or multi-turn), extraction methods (half or third integer, chromatic or achromatic), and operation parameters (machine tune and chromaticity).

\section{Introduction}

The recent report of the NSAC Subcommittee on Electromagnetic Interactions 1 specified the beam requirements for a high duty factor, multi-GeV, electron machine which were felt to represent necessary and sufficient conditions for the realization of the physics program. During the past several years, the Southeastern Universities Research Association (SURA) accelefator physics group has considered several possible accelerator concepts which might achieve the required beam characteristics. Based on this study the linac-pulse stretcher ring design was found to be the superior choice in terms of cost, beam quality, and potential for upgrade to higher energies. The machine design specifications which meet or exceed those specified by the NSAC Subcommittee are given in Table 1 .

Table 1. Accelerator Design Requirements

\begin{tabular}{|c|c|}
\hline Energy Range & $0.5 \leq \mathrm{E}_{\mathrm{o}} \leq 4 \mathrm{GeV}$ \\
\hline Upgrade & $0.5 \leq \mathrm{E}_{\mathrm{O}} \leq 6 \mathrm{GeV}$ \\
\hline Energy Variability & $\begin{array}{l}\text { Continuously from } \\
0.5 \mathrm{GeV}\end{array}$ \\
\hline Energy Spread & $\Delta E_{O} / E_{O} \leq 2 \times 10^{-3}$ \\
\hline Duty Factor & $>80 \%$ \\
\hline Number of Beams & $>2$ \\
\hline I (total) & $240 \mu \mathrm{A}$ \\
\hline Emittance & $\begin{array}{c}0.2 \pi \mathrm{mm}-\mathrm{mr} \text { in one plane } \\
0.4 \pi \mathrm{mm}-\mathrm{mr} \text { in other } \\
\text { plane }\end{array}$ \\
\hline Electron Polarization & $\begin{array}{l}\text { Longitudinal or trans- } \\
\text { verse at all energies }\end{array}$ \\
\hline
\end{tabular}

The basic concept of the linac-pulse stretcher ring is to transform the time structure of a pulsed linac to a high duty factor. The high current (100's $\mathrm{mA})$, short ( $\mathrm{Hs}$ ) linac electron beam pulse is injected into a pulse stretcher ring (PSR) and continuously extracted from the PSR in the time between linac pulses (ms) to produce a high duty factor $(>80 \%)$, intense (100's HA) electron beam. There are several interdependent design parameter choices available in this scheme. In the first part of this paper, the ramifications of these choices will be discussed, and finally, a linac-pulse stretcher design which meets or exceeds the beam characteristics specified in Table 1 will be presented.

\section{Linac Design Options}

The linac design is coupled to the PSR design characteristics. Single turn injection was chosen for the PSR with a circumference of $360 \mathrm{~m}$ which leads to a total linac beam pulse length of approximately 1.2 us. A further specification was given by the desire for an average beam current of $240 \mu \mathrm{A}$. A reduction in the linac cost may be realized if the bcam pulse is recirculated through some of the accelerating sections. To avoid the complications arising from multiple simultaneous beams in a section, only head-to-tail recirculation was considered. Then, the parameter choices remaining are the type of accelerating structure, the number of beam recirculations, the average rf power, and the repetition rate.

$\mathrm{S}$-band (2856 MHz) was chosen as the 1 inac rf frequency because the technology for accelerating systems of this frequency is well known and widely avallable. Further, this frequency will allow for the subharmonic bunching of the linac beam to be compatible with the PSR rf requirements and the need for synchronous injection.

The possible accelerating sections for the linac fall into two general categories; standing wave (SW) and traveling wave (TW). Preliminary considerations of the short pulse lengths of the linac beam indicate that existing designs of SW structures do not exhibit any virtue over a disk-loaded, constant gradient TW guide in terms of achievable gradients or reduction in total $\mathrm{rf}$ energy required of the source for particle acceleration to a particular energy. The increase in required rf energy for the SW structures over that needed by a TW structure, in spite of the higher shunt resistances of the $S W$ cavities, occurs because of the long filling times associated with the high $Q \mathrm{SW}$ design. Therefore, a SLAC-type TW accelerating section design was chosen.

A primary consideration in any accelerator design is cost, and therefore, the total cost including the initial cost of the linac and recirculation path(s) and the 10 year $A C$ power cost was evaluated as a function of the available parameters within the range dictated by technology and accelerator physics. Preliminary analysis showed that maximum economy was achieved with one $40 \mathrm{MW}$ klystron driving one SLAC-type TW accelerating scction, and therefore, this unit was used as the basic building block for the linac.

A linac with head-to-tail recirculation(s) may have some sections through which the beam passes only once and some through which the beam is recirculated. It was found that maximum economy was obtained when the beam was recirculated through the maximum number of sections possible. The path length requirements of a head-to-tail, achromatic, and isochronal recirculation of a $1.2 \mathrm{\mu s}$ beam pulse set an upper limit of approximately 35 sections through which the beam may be recirculated.

The total cost versus the number of recirculations based on designs which utilized the maximum number of recirculated sections was evaluated. As can be seen in

* Work supported by the University of Virginia and the Southeastern Unfversities Research Association (SURA). 
Figure 1, a distinct savings is realized by a single recirculation over that of an unrecirculated linac. The savings arc less dramatic for additional recirculations, and the total cost actually increases at four recirculations due to the cost of additional recirculator paths. A beam break up current threshold of about $420 \mathrm{~mA}$ was estimated by computer simulation 2 for the case of a single recirculation. This limit is reduced to approximately $300 \mathrm{~mA}$ for a two recirculation machine. In addition, the rf pulse length and therefore, the duty factor increase by about $40 \%$ in going from one to two recirculations. Because of the reduced beam break up current threshold, increased average if power requirements, and the minimal cost reduction, the single pass recirculation is consicered to be near the optimum choice.

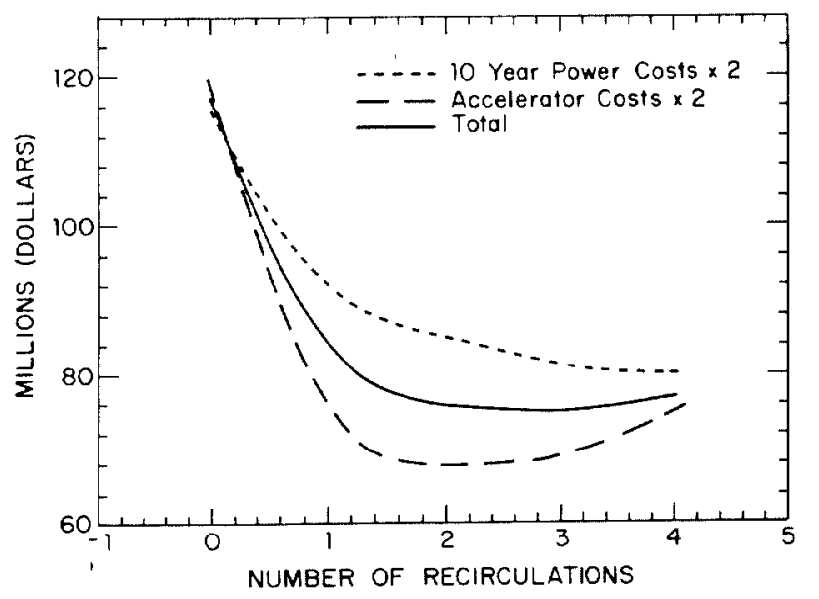

Figure 1. Total Cost vs. Number of Recirculations.

The final linac design parameter remaining is the repetition rate. There is a weak cost dependence on this parameter arising primarily from the AC power costs. The $A C$ power costs were found to increase about $13 \%$ in going from a repetition rate of $720 \mathrm{~Hz}$ to 1000 $\mathrm{Hz}$. The lower repetition rates require less average $\mathrm{rf}$ power which may be an advantage from the standpoint of available technology. On the other hand, given the same average current, the lower repetition rates result in a higher peak current in the linac which increases the possibility of beam break up phenomena. There will also be higher currents and longer store times in the pulse stretcher ring both of which will exacerbate possible instability problems. Fortunately, however, the linac can be designed for operation at $1000 \mathrm{~Hz}$ without precluding the option of a reduced repetition rate should that prove desirable.

\section{Pulse Stretcher Ring (PSR) Design Options}

The choices to be made in designing a PSR are a) single or multi-turn injection in the horizontal or vertical plane, b) half or third integer resonant chromatic or achromatic extraction, c) the total machine circumference, d) the $\mathrm{rf}$ frequency, and e) the machine tune and chromaticity. The discussion of these parameters given below is in many ways very specific to the design of a PSR for this facility. The final choices given may not be particularly optimal either in terms of cost or performance for a PSR operating in a different regime. Unlike the linac, virtually all of the parameters of the PSR were chosen on the basis of extracted beam quality and beam extraction control as opposed to cost.

The extraction may be accomplished chromatically or achromatically. With chromatic extraction, the beam energy is brought down to the resonance energy by the loss of energy through synchrotron radiation. Because the relative energy loss due to synchrotron radiation goes as the third power of the energy, the rate at which the beam will lose energy, and therefore, be extracted will have a very large variation over the desired energy range of 0.5 to $4 \mathrm{GeV}$. Chromatic extraction is still possible, however, if wigglers are employed to enhance the energy loss rate at the lower energies. The magnetic bend radius wnuld then he chosen to provide the desired loss rate at the higher energies and the injected energy spread could be adjusted accordingly over the nid energy range to accomplish the total beam extraction in the time between linac pulses. The requirement of wiggler magnets for the lower energy extraction would increase the cost and complexity of operation. In the higher energy range where the energy loss would be realized through synchrotron radiation from the dipoles alone, there would be no parameters to adjust with the exception of the injected beam energy spread. Of course, the resonance producing magnetic elements could also be varied to some degree to provide additional control of the extraction procedure, although the extraction would then no longer be strictly chromatic. While this could almost certain$1 y$ be accomplished, the increased complexity and loss of extraction control does not favor chromatic extraction for this particular application.

With achromatic extraction, the beam is injected and maintained at a particular energy with an $r f$ system. Resonant extraction is accomplished by adjusting multipoje elements such that the stable phase space goes to zero in such a way that the total stored beam is extracted in a smooth and continuous fashion in the time between linac pulses. The primary virtue of this scheme is that the rate of change of and values of the resonance producing multipole elements are free parameters. They may be adjusted such that the extraction may proceed over periods longer than that dictated by the time between linac pulses, thus creating a more flexible environment. More importantly, a feed back system between extracted beam measurement devices and the multipole resonance producing elements can be used to obtain an extracted beam current which is more constant in time than would otherwise be obtainable. A1though, as mentioned above, a sfmilar feedback system is also possible with quasi chromatic extraction.

With achromatic extraction, the extracted beam energy spectrum will equal that of the stored PSR beam. However, as discussed in more detail elsewhere ${ }^{3}$, the energy spread may be reduced by selective timing of the linac klystrons, the use of an energy compression system, 4-6 or by energy compression in the PSR itself. In the present application, selective timing of the linac klystrons alone will provide an extracted energy spectrum which will meet the design specifications.

Once achromatic extraction has been chosen, the circumference of the ring is, within narrow limits, also determined. The rf power requirements go as the inverse power of the magnetic bend radius. Therefore, it is advantageous to make the bend radius as large as possible. Coupling this requirement with the desire to have a PSR which may be upgraded easily to $6 \mathrm{GeV}$, a magnetic bend radius of $27 \mathrm{~m}$ and a dipole field at $6 \mathrm{GeV}$ of $7.5 \mathrm{kG}$ were chosen. As is evident from the facility layout shown in Figure 2, it is advantageous to have both the extraction and injection on the same side of the ring. The PSR circumference is also related to the linac pulse length. However, in this particular application the PSR lattice space requirements are the determining factor. Then, with the length of the straight sections dictated by the injection and extraction both on the same side of the ring and rf cavity requirements, a total circumference of approximately $360 \mathrm{~m}$ is arrived at. 


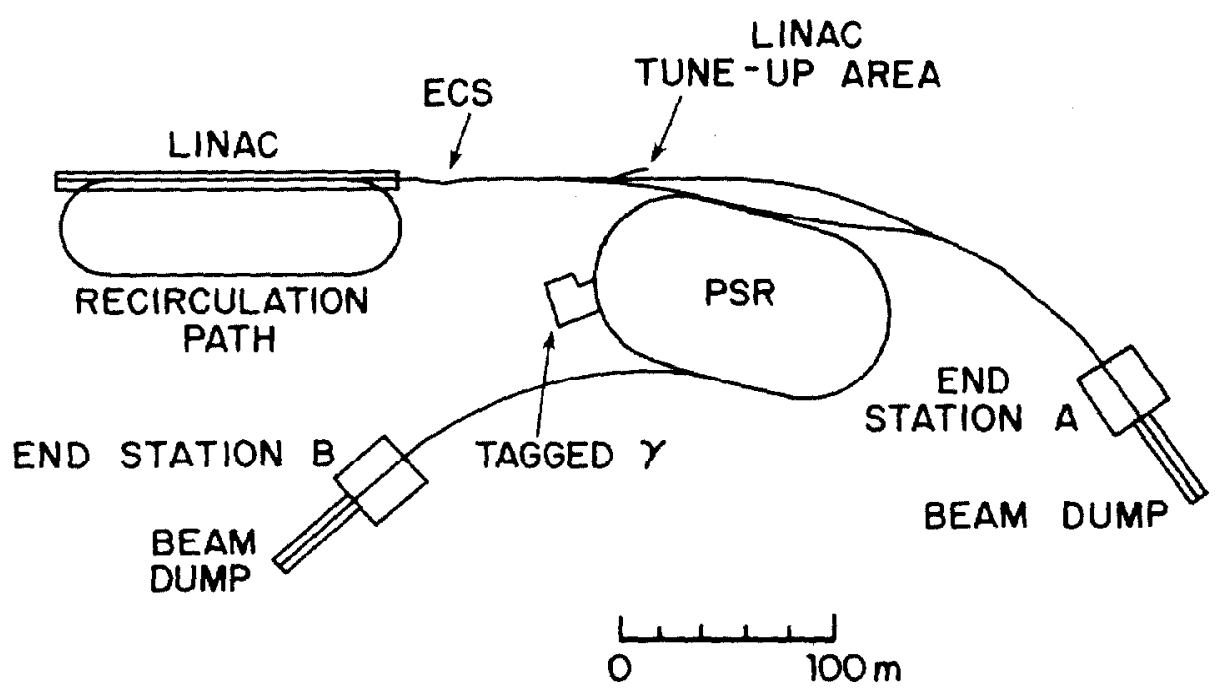

Figure 2, SURA $4 \mathrm{GeV}$ Accelerator Facility Layout.

Either single or multi-turn injection in the horizontal or vertical plane are possible. Because a circumference of approximately $360 \mathrm{~m}$ has been chosen, multi-turn injection would require the linac beam pulse to be longer creating a less efficient, lightly loaded accelerator while dramatically increasing the average if power requirements. Therefore, multi-turn injection would place more stringent requirements on the linac. If multi-turn injection were to be used, then horizontal injection would also be required so that each orbit can be displaced such that the septum is not intercepted. Any $j$ itter in the kickers required for this displacement will then be directly coupled to the horizontal extraction and cause a degradation in the extracted beam emittance and time structure. There will necessarily be a pair of kickers required to compensate for their finite fall time. If horizontal injection is used and the two kickers are not separated by a $\pi$ phase shift, then a residual horizontal offset will remain which would adversely effect the extraction procedure. Further, a multiturn injection scheme would necessarily result in more than one simultaneous, off-axis beams being present in the PSR. This would clearly exacerbate all potential instability problems. If, however, vertical injection is used, then any jitter associated with the injection process will cause only a moderate increase in the vertical beam emittance and not affect the horizontal extraction procedure. For these reasons, single turn, vertical injection was chosen for the PSR.

The possible PSR rf frequency must be a subharmonic of the linac rf frequency of $2856 \mathrm{MHz}$ to enable synchronous injection. The primary considerations are shunt impedance, aperture, and higher order parasitic modes which may cause beam instabilities. A lower frequency cavity would have a larger aperture, but would also have a smaller shunt impedance and more possible narrow band resonance peaks which would potentially need to be damped. Further, the lower frequencies would require a larger number of particles per bunch and create a time micro-structure which would be more evident to the experimenters. The higher frequencies would have a reduced aperturc, but would have a higher shunt impedance, and fewer resonance peaks with the vacuum pipe cutoff frequency being somewhere near $3 \mathrm{GHz}$. A PSR rf frequency of $714 \mathrm{MHz}$ was chosen. This choice limits the number of particles in a microbunch to a reasonable $10^{9}$ particles and permits an adequate aperture of $9 \mathrm{~cm}$ with a practical shunt im- pedance of $15 \mathrm{M} / \mathrm{m}$. Further, the rf fundamental frequency is sufficiently high to limit the number of potentially problematic narrow band resonances.

The resonant extraction may proceed via a thirdor half-integer resonance. While the third integer resonance is not as strong and may proceed more smooth$1 y$, there is no finite stop band. To ensure complete extraction efficiency, either the beam phase space must be "hollow" or the machine tune must be held precisely at the third integer resonance for the time required for the final particles to be extracted. The use of $\mathrm{rf}$ in the ring makes the maintenance of the hollow phase space difficult, and the tolerance requirements on the magnetic elements would become quite high if the tune were required to be maintained precisely for periods of tens of revolution times. Even worse, the beam environment interactions will cause both coherent and incoherent tune shifts which will cause tune fluctuations independent of how precisely the lattice element values are maintained. On the other hand, the half-integer resonance has a finite stop band and hence, the requirement of holding a particular tune for some period of time is eliminated. Therefore, the half-interger resonance was chosen for extraction.

The machine tune and chromaticity are the final parameters remaining to be specified. To eliminate the possibility of the "head tail" instability", the chromaticity is corrected to zero or slightly positive. The fractional part of the tune is dictated for the horizontal plane by the decision to use half integer resonant extraction. For the vertical plane it should be variable over a reasonable range to avoid possible coupling and depolarizing resonances. The integer part of the tune comes from the bending arcs, the required number of straight sections, and the desire to keep the beta functions nearly constant around the PSR. Each arc is composed of $2 \mathrm{I}$ cells matched to the straight sections at either end by a dispersion matching "missing magnet" region. The 2I cells are optical achromats 8 with a betatron tune advance per unit cell of 0.25 in both planes. Such a scheme allows families of sextupoles to be separated by betatron tune shifts of 0.5 in both planes. This sextupole placement permits the control of the chromatic properties of the PSR without the introduction of achromatic effects. Multipoles in the achromatic straight sections introduce achromatic but not chromatic effects. Therefore, the extraction optics may be controlled by 
two sets of independent parameters. Given the design for each arc, the necessary straight sections, and the desire for horizontal half integer resonant extraction, the machine tune is then specified within a narrow. range.

\section{SURA 4 GeV Linac-Pulse Stretcher Design}

The analysis upon which the above discussion is based has lead to the design of a linac-pulse stretcher whose electron beam qualities will meet or exceed thbse raquirements specified in Table 1 . The facility layout is shown in Figure 2 .

The linac has been designed as the source of electron beams which match the admittance properties of the PSR. The linac beams are composed of $1.2 \mathrm{Hs}$ current pulses containing $240 \mathrm{nC} / \mathrm{pulse}$ at a repetition rate of $1 \mathrm{kHz}$ and a mean energy variable from 0.5 to $>4 \mathrm{GeV}$. The linac delivers beam pulses either to the PSR or directly to one of the end stations. Beams of different energies, charge/pulse, pulse width, and repetition rate may be interlaced to provide variation of the individual beams. The Iinac beam characteristics are given in Table 2.

Table 2. Linac Beam Characteristics

$\begin{array}{ll}\text { Energy } & 0.5<\mathrm{E}_{\mathrm{O}}<4.15 \mathrm{GeV} \\ \text { Upgrade } & 0.5<\mathrm{E}_{\mathrm{O}}<6 \mathrm{GeV} \\ \text { Energy Spread } & \Delta \mathrm{E}_{\mathrm{O}} / \mathrm{E}_{\mathrm{O}} \leq 0.2 \% \\ \text { Pulse Repetition Rate } & 1 \mathrm{kHz}(\text { variable) } \\ \text { Bunching Frequency } & 714 \mathrm{MHz} \\ \text { Transverse Emittance } & 5 \pi \times 10^{-3}(\mathrm{MeV} / \mathrm{c})-\mathrm{cm} \\ \begin{array}{l}\text { Number of Interlaced } \\ \text { Beams }\end{array} & 3 \\ \text { Peak Current } & \geq 200 \mathrm{~mA}\end{array}$

Figure 3 outlines the general plan of the linac. Electrons are generated, polarized if desired, bunched and preaccelerated to $35 \mathrm{MeV}$ in the injector region. The accelerating structures consist of SLAC-type, constant gradient, traveling-wave (TW) sections. Each section is fed with a pulsed, $40 \mathrm{MW}, 2856 \mathrm{MHz}$ klystron having an rf pulse length of $3.2 \mathrm{\mu s}$. The design provides for acceleration through 5 such sections before entrance into a 35 section region in common with a single pass, head-to-tail beam pulse recirculation. Thus, $4 \mathrm{GeV}$ electrons are produced by acceleration through effectively a 75 section machine.

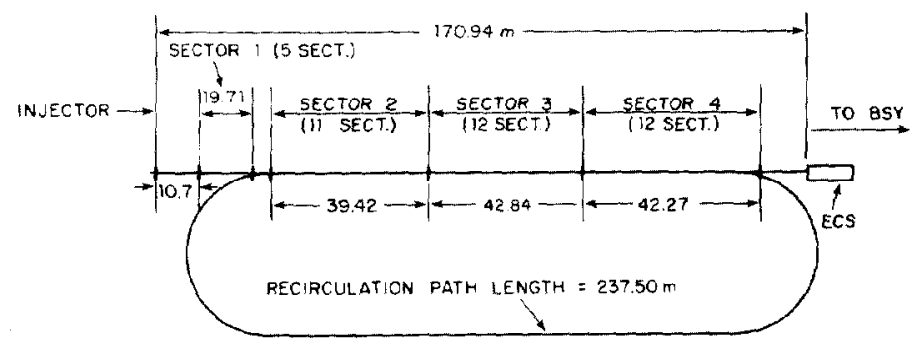

Figure 3. Iinac Layout.

For linac beam transport, a system of alternating quadrupole singlets with a center to center spacing of approximately $6.6 \mathrm{~m}$ has been designed. A computer simulation of beam break up phenomena was done assum- ing this focusing system and two types of SLAC cavities with their HEM 11 mode frequencies shifted by $2 \mathrm{MHz}$ relative to each other between the two cavity types.10 The study indicated a beam break up current threshold of $720 \mathrm{~mA}$ and $420 \mathrm{~mA}$ for the unrecirculated and recirculated beam respectively.

The linac energy may be easily upgraded to $6 \mathrm{GeV}$ by the addition of 37 accelerating sections and $k 1 y-$ strons. It is possible to place approximately 30 of these in the achromatic straight section of the recirculation path. Those remaining would be placed in the present injector region and the injector would then be moved upstream.

The recirculator chicane consists of two 180 degree achromatic arcs separated by a straight, achromatic phase matching section. The recirculator transport has been designed such that only an increased electrical drive will be required to complete an upgrade to $6 \mathrm{GeV}$.

The beam switch yard (BSY) provides transport of electron beams from the linac to the PSR or to end station A directly. In addition, transport of beams extracted from the PSR to end stations $A$ and $B$ is provided.

Flexibility has been built into the design to allow for future expansion. Drift sections are provided at appropriate locations to allow insertion of elements necessary for additional channels. The present design explicitly provides for two additional end stations in the area of the proposed end station $A$ and an additional injection line for other electron rings. The magnetic element parameters of the BSY were chosen so that only increased electrical drive will be required to enable the transport of $6 \mathrm{GeV}$ electrons.

An energy compression system (ECS) ${ }^{4-6}$ has been placed at the entrance of the BSY. This system will be used for beam energies of $\leq 2 \mathrm{GeV}$ if the energy attainable by selective timing of the linac klystrons is not sufficiently small for particular applications. The beam will enter and exit the achromatic, anisochronal ECS magnet chicane through the action of pulsed dipoles. Therefore, the use of the ECS for a particular energy will not preclude the availability of beams of other energies on a pulse to pulse basis. The magnetic elements of the BSY up to the point of the PSR injection channel are pulsed, and therefore, provide the capability of pulse to pulse energy variation of the linac beam.

Transverse polarization will be required in the PSR. However, in most instances longitudinal polarization is necessary at the target position of the end stations. Therefore, there will be a polarization rotation chicane in the PSR to end station lines to rotate the transversely polarized electrons extracted from the PSR to Iongitudinally polarized electrons over the energy range of 0.5 to $4 \mathrm{GeV}$ with nearly $100 \%$ efficiency. The theory of this lattice is described in dctail elsewhere in these proceedings. 11

Single turn, vertical injection into the PSR is used. All machine elements of the facility are in a single plane, and therefore, the vertical injection is accomplished by an achromatic chicane in the BSY which brings the beam up out of the common plane and injects it vertically from above into the PSR at angle of approximately $4^{\circ}$ relative to the horizontal. Magnetic and electrostatic elements in the PSR injection region return the $4^{\circ}$ injection slope to horizontal in the PSR.

The PSR lattice is shown in Figure 4, and the primary design parameters are given in Table 3. Synchronous injection of the linac beam pulse is accomplished by single turn, vertical injection on axis in both planes. There are two extraction insertions one on either side of the ring, and the total PSR current of $240 \mu \mathrm{A}$ may be partitioned arbitrarily between these two extraction channels. The beam may be further separated in the BSY, and space has been provided in 


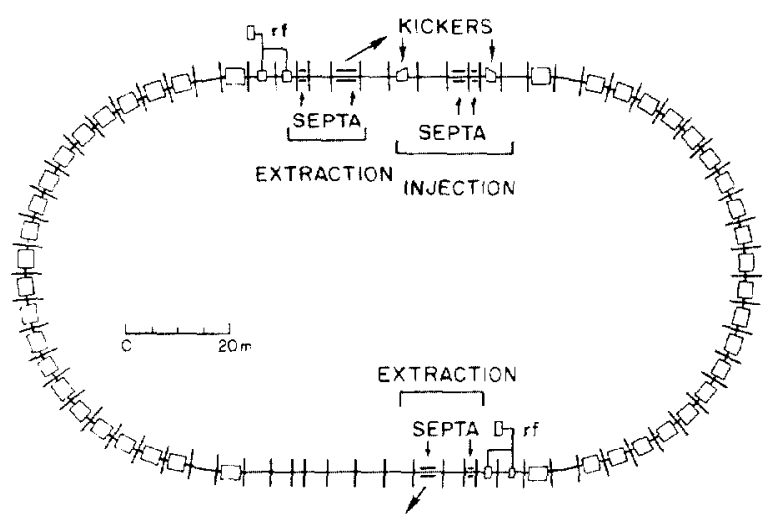

Figure 4. Pulse Stretcher Ring Layout.

the BSY to end station A to provide for the implementation of at least two additional end stations in this region. The tagged photon facility is directly adjacent to the PSR. A modified PSR lattice dipole in conjunction with a very thin radiator target is used to produce tagged photons for this facility.

Table 3. Pulse Stretcher Ring Parameters

Extracted Beam Parameters:

$\begin{array}{ll}\text { Energy } & 0.5<\mathrm{E}_{\mathrm{O}}<4 \mathrm{GeV} \\ \text { Upgrade } & 0.5<\mathrm{E}_{\mathrm{O}}<6 \mathrm{GeV} \\ \text { Vertiral Emittance } & 0.1 \pi \mathrm{mm}-\mathrm{mr} \\ \text { Horizontal Emittance } & 0.3 \pi \mathrm{mm}-\mathrm{mr} \\ \text { Total Current } & \leq 240 \mu \mathrm{A} \\ \text { Duty Factor } & >90 \% \\ \text { Circumference } & 362.773 \mathrm{~m}\end{array}$

General Parameters:

Magnetic Radius

$26.855 \mathrm{~m}$

Harmonic Number

Momentum Compaction

0.022

Betatron Tunes:

Horizontal

8.5 (on resonance)

Vertica1

8.8 (variable)

Chromaticity

(corrected/uncorrected)

$\begin{array}{ll}\text { Horizontal } & -10.4 / 0 \\ \text { Vertical } & -11.4 / 0\end{array}$

RF System:

Frequency

$714 \mathrm{MHz}$

Peak Voltage

(variable/continuous)

$4.5 / 1.5 \mathrm{MV}$

Average Power

(variable/ continuous)
The beam extraction is begun by a $1.6 \mathrm{~m}$ electrostatic septum. 12 The extraction septum is located about $1.5 \mathrm{~cm}$ from the closed orbit where the pitch of the extraction trajectories is approximately $6 \mathrm{~mm}$. A magnetic septum is used to complete the $4^{\circ}$ horizontal extraction from the PSR.

The PSR rf power is provided by two systems; one on continuously and the other operational only during the first several $\mu$ s after the linac beam injection. Upon injection of the linac beam, both systems are operational providing an overvoltage of approximately 6. Due to the momentum compaction of the PSR lattice, the injected energy spread and $r f$ phase length are rotated in the rf bucket to yield a smaller energy spread and larger rf phase length. At this time, the variable $r f$ phase is turned off reducing the overvoltage to approximately 1.2. Simulations of this procedure predict a factor of four decrease in the PSR beam energy spread resulting from this procedure. A more detailed account of this system may be found elsewhere. ${ }^{3}$

An investigation of the possible PSR instabilities was done. The primary potentially current limiting instabilities were found to come from the possible narrow band resonances originating in the rf cavities. However, the $\mathrm{rf}$ frequency of $714 \mathrm{MHz}$ is sufficiently high that only a few impedance peaks will exist and it is felt that these can adequately be selectively damped. As a precautionary measure present plans include the installation of a higher harmonic cavity to create an additional damping mechanism.

The PSR circumference of $360 \mathrm{~m}$ could be reduced somewhat if no energy increase were planned. However, the magnetic lattice parameters are chosen so that the PSR may be easily upgraded in energy. The PSR energy range may be increased to $6 \mathrm{GeV}$ by the addition of $r f$ power and increased electrical drive to the magnetic lattice elements.

\section{References}

1. Report of the NSAC Subcommittee on Electromagnetic Interactions, sponsored by DOE and NSF and chaired by Peter Barnes from Carnegie-Mellon University, 1982 .

2. A computer code which models cumulative beam break up in a linac was obtained from $R$. Helm of SLAC and modified to include recirculation.

3. "Proposal for a National Electron Accelerator Laboratory", Southeastern Universities Research Association, Oct. 1982.

4. H. Herminghaus et a1., Nuc1. Instrum. Methods 113 , 189 (1973).

5. M. Sugawara et a1., Nuc1. Instrum. Methods 153, 343 (1978).

6. J.C. Sheppard et al., Proc. 1981 Iinear Acc. Conf., $266(1981)$

7. C. Pellegrini, Nuovo Cimento, 64A, 477 (1969).

8. K.I. Brown, "A Second Order Optical Achromat", SLAC-PLB-2257 (Feb. 1979).

9. R.V. Servranckx and K.L. Brown, "Chromatic Corrections for Large Storage Rings", SLAC-PUB-2270 (Feb. 1979).

10. R. Helm, private communication.

11. B.E. Norum and R.C. York, "An Energy Independent Spin Precessor", these proceedings.

12. J. Walton et a1., IEEE Trans. Nuc1, Sci. NS-20 (1975) $109 \overline{1 .}$

External, high duty factor electron beans are produced by achromatic, half-integer resonance extraction of the PSR beam in the horizontal plane. The topology of the stable phase space is controlled by a ramped quadrupole-octupole pair in each straight section. A feedback system between the external beam monitoring devices and the ramped multipoles help to ensure the quality and stability of the extracted bedm current. 